\title{
La fabrication de l'identité : la représentation des conflits internationaux dans les médias
}

\section{Luo Yicheng et Si Jinxin}

\section{(2) OpenEdition}

12 Journals

Édition électronique

URL : http://journals.openedition.org/communicationorganisation/3426

DOI : 10.4000/communicationorganisation.3426

ISSN : $1775-3546$

Éditeur

Presses universitaires de Bordeaux

\section{Édition imprimée}

Date de publication : 1 juin 2006

Pagination : 252-260

ISSN : $1168-5549$

\section{Référence électronique}

Luo Yicheng et Si Jinxin, « La fabrication de l'identité : la représentation des conflits internationaux dans les médias », Communication et organisation [En ligne], 29 | 2006, mis en ligne le 21 juin 2012, consulté le 18 décembre 2020. URL : http://journals.openedition.org/communicationorganisation/ 3426 ; DOI : https://doi.org/10.4000/communicationorganisation.3426

Ce document a été généré automatiquement le 18 décembre 2020.

(C) Presses universitaires de Bordeaux 


\title{
La fabrication de l'identité : la représentation des conflits internationaux dans les médias
}

\author{
Luo Yicheng et Si Jinxin
}

1 Le conflit est un comportement qui existe dans toute communication humaine. Aujourd'hui dans le monde entier, tant sur le plan tant national que local, le conflit est omniprésent sous forme de disputes et d'oppositions culturelles, politiques voire raciales. Les médias et les conflits internationaux sont intimement liés: les conflits attirent les médias et les médias font des reportages sur les conflits. De plus, les médias influencent et soutiennent des conflits parmi lesquels les conflits internationaux, les médias sont traducteurs pour le public et lieu symbolique des luttes entre idéologies différentes. Tous les propos concernant les conflits et les comportements intermédiaires participent non seulement des conflits sont le résultat de la structuration et de l'interaction entre toute une série de logiques médiatiques- mais aussi de la structuration culturelle de la société. La représentation des conflits par les médias est étroitement liée à la culture et à la politique, et influe de manière non négligeable sur la formation et l'évolution de la culture des masses.

2 Pour mieux interpréter le rôle des médias dans la signification des conflits, le texte abordera les conflits internationaux, dont l'importance n'est pas à démontrer, afin de mettre en évidence les comportements, la cible et les moyens d'interprétation des médias ainsi que les relations de pouvoirs dans lesquelles les médias se trouvent. Il mettra en relation la couverture des conflits par les médias et la construction de la conscience sociale; il analysera la construction de l'identité au cours de la représentation médiatique des conflits internationaux, en cherchant les corrélations pouvant exister entre la représentation des conflits dans les médias et l'identification des représentations, entre le processus de représentation de l'identification sur le plan culturel et les relations avec le pouvoir. Le but étant de démontrer que les médias peuvent avoir une action plus positive dans la communication interculturelle. 


\section{L'identification : les conflits internationaux et le motif de la représentation des médias}

3 Les opinions divergent quant aux causes des conflits internationaux. Les " réalistes " déclarent que la raison des conflits internationaux provient des activités de la structure et de la répartition des pouvoirs entre les pays. Les « conflitistes " pensent qu'avec le renouvellement du nationalisme et du fondamentalisme, avec les conflits entre les grandes cultures, les conflits entre les communautés seront de plus en plus violents. Et les «structuralistes" prétendent que les conflits se produisent sur la base de divergences au niveau de l'identification culturelle, du milieu social, de l'intérêt économique et du pouvoir politique. Par ailleurs, l'identification a une signification particulière dans les conflits internationaux. L'identification se fait sur la base de la discrimination et de la violence entre les groupes. Les conflits et la prévention des conflits dépendent non seulement de l'équilibre des forces, mais aussi de la structure intérieure, de la conception de la valeur, de l'identification et de la culture du pays.

4 L'identité désigne non seulement l'identité et l'individualité du comportement d'un corps social, c'est à dire le règlement de la distinction entre le comportement de ce corps social et celui des autres, mais aussi le résultat de la structuration sociale. Comme Calhoun le dit « toutes les langues et les cultures ont les moyens de se distinguer des autres ». Les connaissances sur soi-même sont toujours structurées. L'identification est un processus de structuration des significations sur la base des caractéristiques de la culture ou de celles d'une série de cultures. L'identification est enfin décidée par les conventions de l'organisation et du système de la société. Autrement dit, on connaît sa propre identité en fonction des autres et l'identité existe dans la relation avec autrui.

5 Ce qui est étroitement lié à la relation internationale, c'est l'identité collective. Quand l'identité va au-delà des connaissances de son propre comportement, c'est-à-dire quand on va vers autrui et qu'on met les autres dans la définition de sa propre identité, que l'on rend une identité plus complexe en opérant par cercles concentriques, cette transcendance permet la socialisation de sa propre identité ; ainsi l'identité collective peut se former. L'identité collective sur le plan international est une conception de la «valeur neutre». Elle désigne non seulement l'identification "positive» de son comportement par rapport à l'identité de ses amis, mais aussi l'identification «négative » par rapport à celle de ses ennemis. La relation entre les pays sur la base de l'identité collective peut être sous forme de coopération ou de conflit.

6 Le processus de la structuration de l'identité est un facteur important dans l'apparition et le renforcement des conflits. Dans les relations humaines, les conflits sont causés par l'interprétation libre et la définition individuelle de l'identité. L'identité est en relation intime avec le sens de la sécurité et le contrôle de la sécurité. L'identité est un système du contrôle stressant, elle pourrait renforcer la confiance, la prévoyance et le sens du contrôle. L'identification intervient dans la comparaison avec les autres. L'homme tend à définir son identité à travers la confirmation de sa différence avec les autres. Cette distinction entre autrui et soi-même provoque souvent des conflits. Les différences formées au cours de la structuration de l'identité maintiennent et renforcent les conflits. L'identité est un lien important entre la relation des groupes et les besoins individuels. Si une collectivité permet à un individu d'avoir une identité positive, cet individu cherchera à faire partie de cette collectivité. L'identification sociale et le 
classement de soi-même sont le prélude de la construction des différences de l'identification collective et de la collectivité. (Mitchell, 1981).

7 Le terme de «représentation par les médias » est emprunté au structuralisme. Depuis les années 60 , les penseurs ont remarqué que le monde qui nous entoure, est un monde issu du conditionnement et de la transformation de la culture et qu'il résulte d'une structuration du langage et de la société. Les connaissances ne s'acquièrent pas uniquement au contact de l'environnement extérieur, elles sont structurées positivement par les individus sur la base de la cognition. La processus de cognition interprète et organise le monde vécu. La première caractéristique des médias est d'imaginer authentiquement une certaine relation au monde et ensuite de décrire cette relation dans les médias. Donc, la relation imaginée se transforme en une réalité déformée et restructure le mode de vie et de production des hommes. (PAN Zhichang, 2004). En ce qui concerne les médias, la représentation authentique est une structure relevant d'un langage et d'une société; dans un contexte spécifique individuel et culturel, la représentation correspondante est toujours fondée sur les traditions culturelles ou les conventions morales.

8 La représentation par les médias est en relation étroite avec le thème de l'identification. D'une part, l'identification produit un contexte présupposé à l'objet représenté et ce contexte dépend de la tendance culturelle du réalisateur de la représentation et de son jugement de valeur. L'identification est une position adoptée par le groupe ; elle définit les repères et le cadre spatial, le centre des groupes et des nations dans le système de la société internationale, ainsi que les manières de penser et les règles identitaires. D'autre part, les médias sont les représentants d'une culture donnée en utilisant le système des signes. Les médias produisent non seulement des textes, mais aussi fonctionnent comme une structuration de l'identification à la société et agissent ainsi en relais de l'identification.

\section{La texture culturelle de l'identité de la structuration}

9 Le conflit international est un signe culturel et l'analyse des médias est en ce sens signifiante. Se trouvant dans des contextes idéologiquement différents, les médias structurent le langage, la réalité imaginaire et l'imagination réelle en faisant appel à l'ordre mondial, l'intérêt national et l'identification nationale. Le reportage sur les conflits internationaux est en concurrence avec l'identification nationale. Le système des médias dans chaque pays a sa base historique servant à structurer le langage de l'identification et chaque langage est partiel, essentiel et sert la cause nationale.

10 L'expérience et la tradition ont une grande influence sur la représentation des conflits tels que les couvrent les médias. L'histoire personnelle, la mémoire, l'idéologie, le système des signes, la langue et la géographie interagissent sur le vécu des hommes. La représentation de l'identité, la description des informations peuvent s'opposer, le processus de l'évolution de l'identification se produit dans le temps et l'espace, dans la vie quotidienne. La représentation continue des expériences de la vie courante, la mémoire collective, l'ordre symbolique et l'auto imagination constituent la signification de la vie sociale, les références et le contexte des interactions entre les hommes. Lorsque le conflit éclate, on fait appel aux connaissances et aux expériences vécues dans son passé. 
11 La représentation du conflit est fondée sur la structuration du langage issu de sa propre culture. Elle est caractérisée par l'affirmation de soi, se manifeste à travers une supériorité nationale. On a tendance à valoriser son propre mode de vie et à dévaloriser celui des autres. On pense toujours que le groupe dont on fait partie est supérieur à celui des autres.

12 Le renforcement de l'identité se structure en comparaison avec autrui. L'opposition et la division peuvent renforcer ce genre d'identification. Les discours tenus justifient et glorifient souvent son propre camp et dénigre celui des autres. Par exemple, dans la description et la structuration sociale que l'on trouve dans les pays nationalistes, les propos souvent utilisés portent exclusivement sur le racisme, la purification des races, la souveraineté nationale afin de légitimer la violence rencontrée dans ces groupes. L'identité liée à la souveraineté nationale peut être renforcée par des encouragements, par un discours vantant la gloire historique, les mérites réels afin de mobiliser les individus dans le conflit. Comme David-Morris·Kevin·Robin le dit, l'écran n'est pas seulement un intermédiaire pour nous montrer les images mais c'est aussi un moyen de projeter la crainte, l'illusion et l'envie chez les autres.

13 Dans les pays nationalistes, on voit le plus souvent se construire une identité fondée sur des valeurs telles que la légalité et l'exclusivité. Benedict Anderson définit la nation comme une communauté politique imaginaire qui possède la même origine et la même souveraineté. Les nations et l'identité nationale transcendent le temps et l'espace et s'interpénètrent. La souveraineté, les droits du citoyen et le nationalisme sont étroitement liés. Le nationalisme a beaucoup de caractéristiques interactives: les mêmes bases politiques entre pays nationalistes, la description de la souveraineté du pays, les caractéristiques idéologiques liées à la classe dominante, l'attitude et la psychologie reposant sur le couple ciyoyen-nation et la mémoire de la force symbolique de la communauté qui fabrique l'imagination. La nation forme un tout qui se fonde sur l'histoire et la culture imaginaires. Au cours des conflits, les éléments ci-dessus sont mis en valeur pour résister aux autres.

Dans les conflits, la nation renvoie à la mémoire du passé et à la mobilisation du peuple. Beaucoup de conflits après la guerre Froide se trouvent relayés par des discours faisant état de l'origine des nations. Le territoire des ancêtres est ressuscité pour légitimer les conflits présents et futurs.

15 Au cours de la structuration de l'Etat d'Israël, de la Palestine et de la Serbie, on a ressuscité le passé lointain en mettant en avant les ancêtres pour trouver des arguments de poids, distinctifs par rapport aux autres.

\section{Le processus du pouvoir de la fabrication de l'identité}

Fouquet croit que la représentation n'est pas seulement une exploration et une interprétation pour signifier, mais aussi une production intellectuelle associée à un langage spécifique. Dans le système et l'histoire définis par un temps et un espace spécifique, la représentation s'appuie sur des techniques spécifiques et sur une stratégie particulière. Ainsi la relation de pouvoirs intervient dans la production de ces connaissances. (Stuart Hall, 2003). Les études sur la représentation des conflits doivent prendre en compte le système politique dans un contexte historique spécifique. Selon la pensée de Fouquet sur les connaissances et le pouvoir, on pourrait découvrir que 
l'identification est généralement obtenue par la domination et le contrôle de la structuration, l'avantage de la description relative à l'identification dépend du degré de contrôle des organisations sur les médias. C'est toujours les critères sociaux, l'ordre symbolique et la structure qui dominent dans la société et qui sont des éléments déterminants.

Dans les conflits, les groupes sociaux dominants transmettent leurs valeurs identitaires et distinctives au nom de la nation, de l'intérêt national et d'un objectif légal. L'intérêt national, la sécurité nationale, la souveraineté nationale et la décision nationale en sont les maîtres-mots. Ces mêmes arguments sont repris par les médias qui mettent en relation les classes et les races différentes pour former l'identité collective et maximiser l'ordre de la puissance collective et individuelle.

La représentation des conflits par les medias est d'abord le résultat de la domination idéologique. Les médias respectent les règles culturelles dominantes, existent dans le cadre approuvé par la masse et l'opinion publique. Les médias s'adaptent à cette opinion commune et s'en font le relais. Les médias soutiennent et transmettent les valeurs qui sont celles des autorités en place quand ils véhiculent les signes qui symbolisent la culture relative aux intérêts nationaux. Les conflits internationaux sont plein d'inattendus et ils ne sont compréhensibles que dans un cadre normal, prévisible et ordinaire. En les transformant en connaissances acceptables -bien que les pays occidentaux ne reconnaissent pas que leur opinion commune soit marquée idéologiquement-, les propos concernant les conflits reflètent l'idéologie de leur pays. Chaque fois qu'il se passe des événements, les groupes nationalistes mobilisent les ressources du public, affichent les valeurs-clés de la société pour consolider le prestige de la structure au pouvoir.

19 Le système de la représentation des conflits dans les médias dépend aussi de l'ouverture des intérêts nationaux. Les techniques des médias n'ont pas affaibli l'idéologie nationale, bien au contraire, elles la renforcent. La circulation des informations libres ne changerait pas l'idéologie et la position des dirigeants, des diplomates, des militants et des médias dans les conflits internationaux.

Comme l'ordre international est souvent plus complexe que l'ordre national, on souscrit davantage au principe de mettre en avant les intérêts nationaux dans les conflits internationaux. "les informations internationales sont toujours basées sur l'idéologie nationale même dans un contexte de mondialisation, il y a des informations qui sont diffusées en respectant la position du gouvernement et de l'Etat. Comme toute affaire n'est compréhensible que dans un contexte de cause à effet, les médias y établissent une certaine relation en sélectionnant les faits en fonction de la culture et de l'opinion nationale dominante. » (LI Jinquan, 2004).

21 Non seulement on peut constater que l'idéologie dominante est intégrée à la représentation médiatique des conflits et se fonde sur l'intérêt national, mais aussi que l'opinion commune des élites, à l'intérieur du système établi, renforce cette idéologie. Les dirigeants de l'opinion et les élites du pouvoir structurent le noyau des conflits dans les médias. Quand le pays connait un conflit avec l'extérieur, les élites au pouvoir craignent un bouleversement du thème national. En réaction, les médias le rendent légal et maintiennent le thème enraciné dans l'ordre des privilèges et du pouvoir en place. Comme Hall le dit « les organes des médias ne sont pas toujours directement menacés ou censurés, et ils se plaisent à flatter le pouvoir politique». Les médias diffusent des informations concernant les chefs d'Etats et les diplomates qui profitent 
aux autorités politiques. Ils participent à la structuration de la description des élites nationales devant l'opinion publique et à la légitimation des efforts réalisés. Ils visent à défendre la politique de l'Etat. Les médias utilisent toutes sortes de figures comme des métaphores, des logiques pour décrire les conflits internationaux. Même dans les compétitions pour communiquer les informations internationales. En cherchant les raisons sous toutes leurs formes dans la réalité, les médias tendent à légitimer et défendre les intérêts nationaux.

\section{Conclusion}

L'identité est la base des interactions entre violence et discrimination à l'égard des groupes. La communication met en relation l'identité et le conflit par la structuration et le dialogue ; elle dissimule la logique intérieure des conflits et les contradictions en s'appuyant sur l'identification et sur la structuration culturelle. Notre vécu se construit sur l'histoire personnelle, la mémoire, l'idéologie, le système des signes, la langue et la géographie. Les médias structurent leurs discours sur les conflits via les compétences et les connaissances qu'ils puisent dans le vécu, les valeurs nationalistes et l'imaginaire de l'héroïsme ; la représentation des conflits se manifeste par le contrôle du langage qui se calque sur l'identité et la structuration dominantes. La défense des intérêts nationaux, les élites du pouvoir et les dirigeants de l'opinion s'expriment à l'unisson quand il s'agit de conflits. La représentation des conflits internationaux dans les médias devrait s'affranchir des frontières des connaissances et établir une communauté d'identité plus vaste et plus positive et par là transcender les expériences quotidiennes et l'imagination de la culture nationaliste. $\mathrm{Ce}$, dans le but de construire un cercle concentrique d'intérêts qui permettrait le développement de toute la société internationale.

\section{BIBLIOGRAPHIE}

Susan Carruthers, Les medias occidentaux et la guerre, Edition de Xinhua, 2002.

LI Jinquan, La transcendance de l'hégémonisme occidental : les medias et la modernité de la culture en Chine, Edition de l'Université d'Oxford a Hongkong, 2004.

Josph Nye , La théorie et l'histoire de l'interprétation des conflits internationaux, Groupe d'Edition Shiji, 2002.

Stuart Hall, La représentation : la représentation de la culture et la pratique du message, Edition Shangwu, 2003.

David Moly, Kevin Robbins, L'univers de l'identification : les medias universels, le panorama du monde électrique et les frontières culturels, Edition de l'université de Nanjing, 2001.

GUO Shuyong, De la paix de la communauté du structuralisme » tiré de L'Europe, $\mathrm{N}^{\circ} 2$ de l'année, 2001. 
Manuel Castells, La force de l'identification, Edition des ouvrages des Sciences sociales, 2003.

LI xiguang, ZHAO Xinshu, La puissance des médias, Edition du journal du sud, 2002.

WENG Xiuqi , « La structuration de l'identification et la mémoire collective -De l'événement de la belle île », Réseaux du journalisme de Taiwan, http://www.jour.nccu.edu.tw , 2003.

PAN Zhichang, La nouvelle idéologie et les medias chinois-un thème avancé de la communication du journalisme dans un nouveau siècle, 2004.

NI Erxiong, «Les facteurs des conflits structuraux dans les relations sino-américaines », http:// www.cc.org.cn.

Ross Howard, Conflict Sensitive Journalism, IMS and IMPACS, Demark, 2003.

Vivienne Jabri, Discourses on Violence : Conflict Analysis Reconsidered, Manchester University Press, 1996.

C.R.Mitchell, Structure of International Conflict, Macmillan, London, 1981.

\section{RÉSUMÉS}

Le conflit international est une des composantes de la nature humaine. La représentation que les médias donnent des conflits internationaux est liée étroitement au contexte culturel et politique et influence de manière non négligeable la formation et l'évolution de la culture des masses. En nous fondant sur la représentation du structuralisme donnée par les médias, le présent article cherche à analyser une culture et une logique pratique, à l'œuvre dans les médias, tout en l'assortissant d'exemples concernant les conflits révélés par les médias et le système structural des signes de la société. Nous sommes en mesure d'affirmer que la représentation des conflits internationaux est en relation avec la construction de l'identité. Dans un contexte dont la culture est déjà donnée, par l'identification de soi-même et la distinction d'avec les autres, les médias maintiennent et promeuvent le pouvoir existant au sein de la société, dissimulent une logique intérieure et mettent en avant une information qui fait appel à l'expérience de la vie quotidienne et à la mémoire nationale.

INDEX

Mots-clés : conflit international, médias, identité, structuration, pouvoir

\section{AUTEURS}

\section{LUO YICHENG}

Luo Yicheng est Professeur, directeur de l'institut de la communication et du journalisme de l'Université de Wuhan.

\section{SI JINXIN}

Si Jinxin est Aspirant chercheur du doctorat à l'institut de la communication et le journalisme de l'Université de Wuhan. 